\title{
Rechtsprechung
}

\section{Mona Lisa als Etikett, aber nicht als Marke}

Bundespatentgericht, Beschluss vom 9. Dezember 2015 - 26 W (pat) 508/15

Eine nur unwesentlich durch den Schriftzug Monna Lisa veränderte Abbildung des Werkes Mona Lisa von Leonardo da Vinci kann nicht als Wort-/Bildzeichen eingetragen werden. Wort-/Bildzeichen, die von den angesprochenen Verkehrskreisen als übliches Dekor, nicht aber als Unternehmenshinweis wahrgenommen werden, sind nicht eintragungsfähig. (Leitsatz der Redaktion)

\section{Gründe}

\section{I.}

- Das Wort-/Bildzeichen (braun, grün, schwarz, weiß, gelb, grau, rosa) ist am 5. Dezember 2012 unter der Nummer 011400371 zur Eintragung als Gemeinschaftsmarke in das beim Harmonisierungsamt für den Binnenmarkt (HABM) geführte Register für Waren der Klasse 33: Weine angemeldet worden. Nachdem am 23. April 2013 Widerspruch erhoben worden war, nahm die Anmelderin die Gemeinschaftsmarkenanmeldung am 3. Juli 2013 zurück und beantragte ihre Umwandlung in eine deutsche Marke. Dieser Antrag wurde vom HABM genehmigt und ist am 18. Juli 2013 beim Deutschen Patent- und Markenamt (DPMA) eingegangen, in dessen Register das Anmeldezeichen unter der Nummer 302013041291.5 geführt wird.

Mit Beschluss vom 11. Dezember 2014 hat die Markenstelle für Klasse 33 die Anmeldung wegen fehlender Unterscheidungskraft gemäß §§ 125d Abs. 2 und 4, 37 Abs. 1, 8 Abs. 2 Nr. 1 MarkenG zurückgewiesen.

Zur Begründung hat sie ausgeführt, beim Anmeldezeichen handele es sich lediglich um eine Wiedergabe des weltbekannten Gemäldes der Mona Lisa von Leonardo da Vinci. Dieses Bild werde auf einer Vielzahl von Waren im Bereich des allgemeinen Konsums zur blickfangartigen Hervorhebung als Werbemittel genutzt, um die Aufmerksamkeit des Betrachters zu erwecken. Insbesondere bei Flaschenetiketten sei es gängig, künstlerische Darstellungen als Marketinginstrument zu verwenden. Wegen der Bekanntheit dieses Kunstwerkes und der häufigen Verwendung sähen die angesprochenen Verkehrskreise im Anmeldezeichen keinen betrieblichen Herkunftshinweis.

Hiergegen richtet sich die Beschwerde der Anmelderin, mit der sie sinngemäß beantragt,

den Beschluss der Markenstelle für Klasse 33 des Deutschen Patent- und Markenamts vom 11. Dezember 2014 aufzuheben.
Sie ist der Ansicht, es handele sich gerade nicht um eine originalgetreue Wiedergabe des berühmten Gemäldes, weil es durch den in großer Kurrentschrift zentral auf dem Bild aufgebrachten Schriftzug „Monna Lisa“ sowie das unter dem Gemälde platzierte umrandete Rechteck in sehr auffälliger Weise überlagert werde. Durch die vom weltbekannten Gemäldetitel abweichende Schreibweise mit dem doppelten Konsonanten " $\mathrm{n}$ " und der damit verbundenen anderen Aussprache unterscheide sich
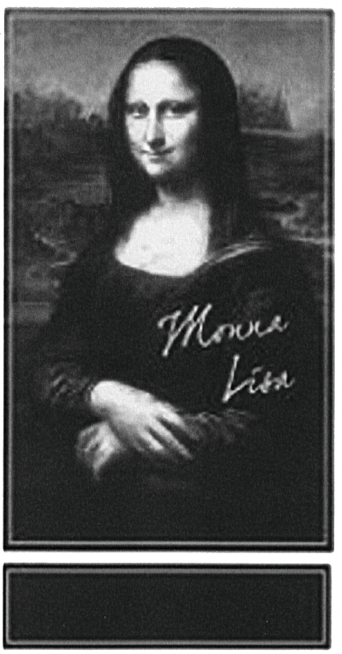

das Anmeldezeichen hinreichend und wirke kreativ und fantasievoll. Denn die angesprochenen Verkehrskreise orientierten sich primär an dem Wortbestandteil "Monna Lisa“, während sie das im Hintergrund platzierte Gemälde allein als Hinweis auf Leonardo da Vinci und damit auf den Sitz der Anmelderin in Vinci auffassten. Der Schriftzug sei auch an einer Stelle angebracht, an der üblicherweise der Name des Getränks, d.h. die Marke, angegeben werde. In der Entscheidung des Bundespatentgerichts - 30 W (pat) 14/12 - David und Venus hätten schon die Auswahl eines Bildausschnitts und die konkrete Aufnahmeperspektive berühmter Kunstwerke Unterscheidungskraft begründet. Ferner werde bei Wort-/Bildmarken dem Wort das entscheidende Gewicht beigemessen, da es in der mündlichen Kommunikation als einziges Element wiedergegeben werden könne. Hinzu komme, dass die deutschen Wortmarken "Picasso" (30705276), "Rubens" (2078822), „MONET“ (885404), „Mona Lisa“ (673340) und "REMBRANDT" (880508) eingetragen worden seien. Auch für die internationale Wortmarke „Monna Lisa“ (699233) sei der Schutz in Deutschland bewilligt worden. Zudem seien Marken wie "Roma" und "Napoleon" für Parfüm oder "Caesars Palace" für Dienstleistungen registriert worden.

Mit gerichtlichem Schreiben vom 13. August 2015 ist die Anmelderin unter Beifügung umfangreicher Recherchebelege darauf hingewiesen worden, dass das angemeldete Wort-/Bildzeichen nicht für schutzfähig erachtet werde. 
In der mündlichen Verhandlung hat die Anmelderin angeregt, die Rechtsbeschwerde zuzulassen, weil "die Interpretation des Gesichtspunkts der Werbeüblichkeit im Rahmen der Beurteilung der Unterscheidungskraft durch das Bundespatentgericht" "nicht im Einklang mit den vom Bundesgerichtshof aufgestellten Maßstäben (Marlene-Dietrich-Bildnis II)“ stehe.

Wegen der weiteren Einzelheiten wird auf den Akteninhalt Bezug genommen.

\section{II.}

Die zulässige Beschwerde ist unbegründet.

1. Der Eintragung des Wort-/Bildzeichens als Marke steht in Bezug auf die Waren der Klasse 33 "Weine" das absolute Schutzhindernis der fehlenden Unterscheidungskraft nach $\S 125 \mathrm{~d}$ Abs. 2 und $4 \mathrm{iVm} \S 8$ Abs. 2 Nr. 1 MarkenG entgegen, sodass die Markenstelle die Anmeldung zu Recht zurückgewiesen hat.

a) Unterscheidungskraft im Sinne des § 8 Abs. 2 Nr. 1 MarkenG ist die einer Marke innewohnende (konkrete) Eignung, vom Verkehr als Unterscheidungsmittel aufgefasst zu werden, das die in Rede stehenden Waren und Dienstleistungen als von einem bestimmten Unternehmen stammend kennzeichnet und diese Waren oder Dienstleistungen somit von denjenigen anderer Unternehmen unterscheidet (EuGH GRUR 2015, 1198, 1201 Rn. 59 f. - Nestlé/Cadbury [Kit Kat]; BGH GRUR 2015, 173, 174 Rn. 15 - for you). Denn die Hauptfunktion der Marke besteht darin, die Ursprungsidentität der gekennzeichneten Waren und Dienstleistungen zu gewährleisten (EuGH GRUR 2010, 228 Rn. 33 - Audi AG/HABM [Vorsprung durch Technik]; BGH GRUR aaO for you). Da allein das Fehlen jeglicher Unterscheidungskraft ein Eintragungshindernis begründet, ist nach der Rechtsprechung des Bundesgerichtshofs ein großzügiger Maßstab anzulegen, sodass jede auch noch so geringe Unterscheidungskraft genügt, um das Schutzhindernis zu überwinden (BGH GRUR aaO - for you). Ebenso ist zu berücksichtigen, dass der Verkehr ein als Marke verwendetes Zeichen in seiner Gesamtheit mit allen seinen Bestandteilen so aufnimmt, wie es ihm entgegentritt, ohne es einer analysierenden Betrachtungsweise zu unterziehen ( $E u G H$ GRUR 2004, 428 Rn.53 - Henkel; BGH GRUR aaO Rn. 16 - for you).

Maßgeblich für die Beurteilung der Unterscheidungskraft zum relevanten Anmeldezeitpunkt (BGH GRUR 2013, 1143, 1144, Rn.15 - Aus Akten werden Fakten) sind einerseits die beanspruchten Waren oder Dienstleistungen und andererseits die Auffassung der beteiligten inländischen Verkehrskreise, wobei auf die Wahrnehmung des Handels und/oder des normal informierten, angemessen aufmerksamen und verständigen Durchschnittsverbrauchers der fraglichen Waren oder Dienstleistungen abzustellen ist (EuGH GRUR 2006, 411 Rn. 24 - Matratzen Concord/ Hukla; BGH GRUR 2014, 376 Rn. 11 - grill meister).
Ausgehend hiervon besitzen Wortzeichen bzw. die Wortelemente von Wort-/Bildmarken dann keine Unterscheidungskraft, wenn ihnen die angesprochenen Verkehrskreise lediglich einen im Vordergrund stehenden beschreibenden Begriffsinhalt zuordnen (EUGH GRUR 2004, 674, Rn. 86 - Postkantoor; BGH GRUR 2012, 270 Rn. 11 - Link economy) oder wenn diese aus gebräuchlichen Wörtern oder Wendungen der deutschen Sprache oder einer geläufigen Fremdsprache bestehen, die - etwa wegen einer entsprechenden Verwendung in der Werbung oder in den Medien - stets nur als solche und nicht als Unterscheidungsmittel verstanden werden (BGH GRUR 2014, 872, 874 Rn. 21 - Gute Laune Drops). Darüber hinaus besitzen keine Unterscheidungskraft vor allem auch Zeichen, die sich auf Umstände beziehen, welche die beanspruchten Waren und Dienstleistungen zwar nicht unmittelbar betreffen, durch die aber ein enger beschreibender Bezug zu diesen hergestellt wird und die sich damit in einer beschreibenden Angabe erschöpfen (BGH GRUR 2014, 1204 Rn. 12 - DüsseldorfCongress).

Entsprechendes gilt für die Beurteilung der Unterscheidungskraft von Bildmarken. Soweit die Elemente eines Bildzeichens nur die typischen Merkmale der beanspruchten Produkte darstellen oder sich in einfachen dekorativen Gestaltungsmitteln erschöpfen, an die sich der Verkehr etwa durch häufige Verwendung gewöhnt hat, wird einem Zeichen im Allgemeinen wegen seines bloß beschreibenden Inhalts die konkrete Eignung fehlen, die mit ihm gekennzeichneten Produkte von denjenigen anderer Herkunft zu unterscheiden. Weist das in Rede stehende Zeichen darüber hinausgehende charakteristische Merkmale auf, in denen der Verkehr einen Hinweis auf die betriebliche Herkunft sieht, kann die Unterscheidungskraft jedoch nicht verneint werden (BGH GRUR 2011, 158, Rn. 8 Hefteinband).

b) Den vorgenannten Anforderungen an die Unterscheidungskraft genügt das Anmeldezeichen in Bezug auf die beanspruchten Waren der Klasse 33 „Weine“ nicht.

aa) Die hier angesprochenen breiten Verkehrskreise - sowohl der Endverbraucher als auch der Weinhandel - werden im Anmeldezeichen ausschließlich die Wiedergabe des weltbekannten Gemäldes der Mona Lisa von Leonardo da Vinci in der typischen Form eines Weinetiketts ohne auffällige Abwandlung und damit nur ein Werbemittel, aber keinen betrieblichen Herkunftshinweis erkennen.

bb) Hauptbestandteil und dominierendes Element des Zeichens ist das Gemälde „Mona Lisa“ des Renaissancemalers Leonardo da Vinci. Daneben verfügt das Zeichen über den Schriftzug "Monna Lisa“ und ein unter dem Gemälde angebrachtes schmales Rechteck in dunkler Farbe.

Bei dem Gemälde handelt es sich um ein sehr berühmtes, möglicherweise sogar das berühmteste Gemälde der Kunstgeschichte. Dies hat auch eine Internetrecherche des Senats ergeben, deren Belege der Anmelderin in der mündlichen Verhand- 
lung übergeben worden sind: Bei einer Bildersuche im Internet auf der Seite www.google.de mit dem Suchbegriff „berühmte Gemälde" erscheint das Gemälde "Mona Lisa“ an erster Stelle. Der Anbieter www.kunstkopie.de, der über 200.000 Gemälde in Form von Kunstdrucken anbietet, führt das Gemälde auf seiner ersten Seite. Laut einem Eintrag unter https://de.wikipedia. org/wiki/Liste_der_teuersten_Gem ist es derzeit mit einem Versicherungswert von 782 Millionen Dollar das wertvollste Gemälde der Welt. Es ist daher davon auszugehen, dass dieses Gemälde in den maßgeblichen Verkehrskreisen nahezu jedermann auch namentlich bekannt ist.

Wie das BPatG in seiner "Mona Lisa"-Entscheidung (24 W (pat) 188/96) bereits 1997 festgestellt hat, handelt es sich bei der "Mona Lisa" um ein sehr häufig und vielfältig eingesetztes, allgemein bekanntes Werbemotiv, auch als Dekor auf Flaschen, durch das die Aufmerksamkeit des Publikums geweckt und letztlich der Kaufanreiz gefördert werden soll. Solche Darstellungen würden vom Verkehr nicht als Hinweis auf die Herkunft der gekennzeichneten Waren aus einem bestimmten Unternehmen verstanden, sondern nur als blickfangartige Hervorhebungsmittel aufgefasst. Das gelte insbesondere für die Wiedergabe eines weltberühmten und dem angesprochenen Publikum ohne weiteres bekannten Kunstwerks, bei dem ein Bezug zu einem bestimmten Warenhersteller oder -händler ohnehin fernliege, sich vielmehr lediglich der genannte Werbeeffekt aufdränge.

Auch die Recherche des Senats hat ergeben, dass eine kunstvolle Gestaltung von Weinetiketten gängiger Brauch bei Weinherstellern ist, worauf die Anmelderin unter Beigabe zahlreicher Belege hingewiesen worden ist. Dabei wird nicht nur auf bestehende Kunst zurückgegriffen, sondern in der Vergangenheit wurden selbst namhafte Künstler wie Marc Chagall, Salvador Dali und Keith Haring mit der Gestaltung von Weinetiketten beauftragt.

Die weiteren Markenbestandteile sind nicht geeignet, den Betrachter von dem dominierenden Eindruck des Gemäldes der „Mona Lisa“ abzulenken und dieses so zu verfremden, dass dem Zeichen Unterscheidungskraft zukommt.

Der Schriftzug „Monna Lisa“ ist nur mäßig groß ausgeführt und in sehr feiner Kurrentschrift gehalten. Er ist wenig auffällig und nicht deutlich lesbar. Die Buchstabenfolge "o-n-n-a" besteht aus sehr eng geschriebenen Buchstaben, so dass bei flüchtiger Betrachtung der Eindruck entstehen kann, es handele sich nur um drei Buchstaben, die dem "M“ folgen, so dass der Betrachter nicht "Monna", sondern "Mona“ liest. Die geringfügig andere Sprechweise mit kurzem "O" fällt daneben nicht maßgeblich ins Gewicht, zumal in manchen deutschen Dialekten auch das "O $\mathrm{O}$ " in "Mona Lisa“ kurz gesprochen wird. Damit kommt dem Schriftzug aber kein weiterer Aussagegehalt zu, als der Betrachter dem Bild sowieso schon beimisst.
"Mona" leitet sich zudem von der italienischen Kurzform "Monna" für "Madonna", also "Frau", her. "Mona“ ist somit nicht der Vorname der dargestellten Person, sondern deren Titel. Mit diesem wurde Lisa als (Ehe-)Frau von Francesco del Giocondo angeredet (https://de.wikipedia.org/wiki/Mona_Lisa; www. leo.org). Die behauptete Verfremdung durch die Abwandlung "Monna" führt somit nicht von dem Gemälde weg, sondern eher noch darauf hin.

Das unter dem Gemälde befindliche Rechteck wird vom Betrachter kaum wahrgenommen. Der Abstand zu dem Gemälde ist gering und der Farb- bzw. Grauton derselbe wie im unteren Teil des Gemäldes. Sollte dieses Rechteck dann noch mit einer Beschriftung versehen werden, wie dies bei zweigeteilten Weinetiketten nach der Recherche des Senats üblich ist, wird der Verkehr dieses Rechteck gar nicht mehr als Zeichenbestandteil wahrnehmen. Aber auch im unbeschrifteten Zustand ist das Rechteck nicht geeignet, dem Anmeldezeichen Unterscheidungskraft zu verleihen. Denn die angesprochenen Verkehrskreise werden ohne weitere Beschriftung den Herstellerhinweis auf der Rückseite der Weinflasche oder am Flaschenhals vermuten. Es konnte nämlich nicht festgestellt werden, dass die Kennzeichnung von Weinflaschen ausschließlich unter Verwendung von Gemälden oder sonstigen künstlerischen Gestaltungen ohne weitere textliche Zusätze, die dann regelmäßig auf den Hersteller hinweisen, gebräuchlich ist.

c) Der normal informierte, angemessen aufmerksame und verständige Durchschnittsverbraucher und der Weinfachhandel werden das Anmeldezeichen somit den Verkehrsgepflogenheiten entsprechend nur als künstlerisch ausgestaltetes Weinetikett, aber nicht als betrieblichen Herkunftshinweis auffassen.

Dies gilt auch und insbesondere unter Berücksichtigung der Entscheidungen des $B G H$ zu "Marlene-Dietrich-Bildnis II" (BGHZ 185, 152 Rn. 21) und „TOOOR!“ (GRUR 2010, 1100 Rn. 28). Danach kommt es darauf an, ob es praktisch bedeutsame und naheliegende Möglichkeiten gibt, das angemeldete Zeichen bei den beanspruchten Waren und Dienstleistungen so zu verwenden, dass die Verkehrskreise es ohne weiteres als Marke verstehen. Bei der Beurteilung der Kennzeichnungsgewohnheiten ist es nach der Entscheidung des EUGH zu „umsäumter Winkel“ (GRUR 2013, 519 Rn. 55 - Deichmann SE) nicht ausgeschlossen, die Prüfung nur auf diejenige Verwendungsmöglichkeit zu erstrecken, die die prüfende Instanz mit Hilfe ihrer Sachkunde auf dem jeweils betroffenen Waren- und/oder Dienstleistungsgebiet als die wahrscheinlichste erkenne. Das setzt allerdings voraus, dass es eine im Vordergrund stehende Verwendungsform gibt und nicht mehrere praktisch bedeutsame Verwendungsformen in Betracht kommen (BGH GRUR 2014, 1204, 1206 Rn. 21 - Düsseldorf(ongress).

Die im Vordergrund stehende, wahrscheinlichste Verwendungsform des Anmeldezeichens auf der beanspruchten Ware "Weine" ist sein Abdruck auf dem Etikett, dessen künstlerische Gestaltung nach den vom Senat festgestellten Kennzeich- 
nungsgewohnheiten in der Weinbranche von den angesprochenen inländischen Verkehrskreisen nur als übliches Dekor, aber nicht als Unternehmenshinweis wahrgenommen wird.

2. Da schon das Schutzhindernis nach $\S 8$ Abs. 2 Nr. 1 MarkenG vorliegt, kann dahinstehen, ob das angemeldete Zeichen darüber hinaus gemäß § 8 Abs. 2 Nr. 2 MarkenG für die fraglichen Waren freihaltungsbedürftig ist.

3. Eine andere Beurteilung ist auch nicht unter Berücksichtigung früherer Entscheidungen und Voreintragungen geboten.

a) Die Entscheidung des BPatG zum Bildzeichen „David und Venus" (30 W (pat) 14/12) ist nicht vergleichbar. Während dort der fotografierte Kopf der berühmten Davidskulptur von Michelangelo und das Gesicht der Venus aus dem weltbekannten Gemälde von Botticelli originell kombiniert wurden, erschöpft sich das verfahrensgegenständliche Wort-/Bildzeichen in der bloßen Wiedergabe eines Originalwerks unter Beifügung geringfügiger und keinesfalls fantasievoller Elemente. Die Schutzfähigkeit des Bildzeichens „David und Venus" wurde ausschließlich aufgrund der konkreten, unverwechselbaren, charakteristischen Komposition angenommen. Dazu gehörte neben der Auswahl des Bildausschnitts und der Aufnahmeperspektive auch die Anordnung der Gesichter zueinander, insbesondere die Art und Weise, wie "David“ zur "Venus" hinauf schaute.

b) Die von der Anmelderin genannten Voreintragungen "Picasso" (30705276), „Rubens“ (2078822), „MONET“ (885404), "Mona Lisa" (673340) und „REMBRANDT" (880508) unterscheiden sich vom vorliegenden Fall schon dadurch, dass es sich ausschließlich um Wortmarken handelt, sodass ein im Vordergrund stehendes Bildelement wie hier fehlt. Ferner wurden sie mit Ausnahme der 1971 für "Weine" eingetragenen Marke „REMBRANDT" entweder nur für Dienstleistungen oder für andere Waren registriert. Im Übrigen liegen sämtliche Eintragungen viel zu lange zurück.

Soweit die Anmelderin anführt, dass für die internationale Marke „Monna Lisa“ (699233) im Jahre 2013 der Schutz für identische Waren in Deutschland bewilligt worden sei, fehlt es schon an der Vergleichbarkeit, weil es sich gleichfalls um eine reine Wortmarke handelt.

Aber selbst wenn diese Marke vergleichbar wäre, könnte es sich um eine rechtswidrig vorgenommene Eintragung handeln. Niemand kann sich auf eine fehlerhafte Rechtsanwendung zugunsten eines anderen berufen, um eine identische Entscheidung zu erlangen (EuGH GRUR 2009, 667, 668 Rn. 18 - Volks. Handy, Volks.Camcorder, Volks.Kredit und SCHWABENPOST). Für die erforderliche Bereinigung des Markenregisters sieht das Gesetz das Löschungsverfahren vor, das von jedermann eingeleitet werden kann.
Bezüglich der von der Anmelderin ins Feld geführten Marken "Roma“" , "Napoleon" oder "Caesars Palace" fehlen Angaben zum Zeitpunkt der Eintragung sowie die Registernummer. Auf jeden Fall fehlt es aber schon nach den Angaben der Anmelderin an Vergleichbarkeit, weil es sich um Wortmarken für andere Waren und Dienstleistungen handelt.

Auch bei den von der Anmelderin in der mündlichen Verhandlung eingeführten neun Voreintragungen für Klasse 33 handelt es sich um reine Wortmarken, die entweder nur aus dem Namen "Mona Lisa" oder aus weiteren Wortelementen bestehen und ebenfalls nicht vergleichbar sind. Die beiden deutschen Voreintragungen "Mona Lisa" stammen von 2004 und 1982 und liegen somit auch schon zu lange zurück. Die übrigen Marken wurden in Dänemark, Frankreich, Italien, Portugal, Spanien oder als internationale Marke eingetragen. Letzterer ist der Schutz in Deutschland verweigert worden. Die im Ausland, in einem anderen Mitgliedstaat der Europäischen Union auf der Grundlage des harmonisierten Markenrechts oder vom Harmonisierungsamt aufgrund der Gemeinschaftsmarkenverordnung getroffenen Entscheidungen über absolute Eintragungshindernisse sind zudem für nachfolgende Verfahren in anderen Mitgliedstaaten unverbindlich (EuGH GRUR 2004, 428, 432, Rn. 63 - Henkel; GRUR 2004, 674 Rn. 43 f. - Postkantoor). Sie vermögen nicht einmal eine Indizwirkung zu entfalten (BGH GRUR 2014, 569, 572 Rn. 30 - HOT; GRUR 2009, 778, 779 Rn.18 - Willkommen im Leben).

4. Da der Senat bei der Beurteilung der Schutzfähigkeit die hierfür von der Rechtsprechung entwickelten Kriterien, insbesondere auch die vom BGH in der Entscheidung zu "MarleneDietrich-Bildnis II“ aufgestellten Maßstäbe angelegt hat, war die Zulassung der von der Anmelderin angeregten Rechtsbeschwerde nicht geboten. Zudem war weder über eine Rechtsfrage von grundsätzlicher Bedeutung zu entscheiden (§ 83 Abs. 2 Nr. 1 MarkenG) noch ist die Zulassung der Rechtsbeschwerde zur Fortbildung des Rechts oder zur Sicherung einer einheitlichen Rechtsprechung als erforderlich zu erachten ( $\$ 83$ Abs. 2 Nr. 2 MarkenG). (Entscheidung von der Redaktion bearbeitet.) 